\section{Avaliação da adequação do rastreamento e diagnóstico de Diabetes Mellitus Gestacional em puérperas atendidas em unidade hospitalar de dois municípios da região do Vale do São Francisco - Nordeste do Brasil}

\section{Evaluation of the adequacy of tracking and diagnosis of gestational Diabetes Mellitus in pregnant women attending a hospital unit in two municipalities in the São Francisco Valley Region- Northeast Brazil}

\begin{abstract}
Objectives: to evaluate the adequacy of tracking and diagnosis of gestational diabetes mellitus in pregnant women attending a hospital unit in two municipalities in the São Francisco Valley region.

Methods: a descriptive study was carried out with 1340 pregnant women who gave birth at the Dom Malan Hospital-IMIP-PE and the Municipal Maternity Hospital of Juazeiro-BA, between April 2011 and January 2012. Women were excluded if they had received a previous diagnosis of diabetes mellitus, if they had not attended prenatal sessions or if their test results were unavailable. Tables of frequency distribution and measures of central tendency and dispersion were drawn up.

Results: the mean gestational age at which a fasting blood sugar level test was requested was 14 weeks and adequate in 46\%. The mean gestational age at which a blood sugar level test was carried out was 16 weeks, less than 30 days after requested in $37.9 \%$; the result was received on average in the 20 th week of pregnancy and this was adequate in $33.4 \%$. A second fasting blood sugar level test was adequately requested and interpreted in $28.1 \%$ of cases; $20.8 \%$ had a fasting blood sugar level equal to or greater to $85 \mathrm{mg} / \mathrm{dL}$ on the first and second test and $11.9 \% \mathrm{had}$ not taken or received the results of the blood sugar test. The tracking/diagnosis was inadequate in $76.3 \%$ $(n=1022)$, and in $5.0 \%(n=67)$ of those who took the Oral Glucose Tolerance Test. Some inadequacy was found in $91.9 \%$ during tracking.

Conclusions: the tracking/diagnosis of gestational diabetes among the pregnant women studied was generally inadequate.
\end{abstract}

Key words Diabetes gestational, Diagnosis, Evaluation
Audimar de Sousa Alves 1

Isabela Coutinho 2

Juliana Conduru Mendes Segatto 3

Lorena Andrade Silva 4

Márcia Danielle de Sousa Silva 5

Leila Katz 6

1,3-5 Universidade Federal do Vale do São Francisco. Petrolina, PE, Brasil.

2,6 Programa de Pós-Graduação Stricto Sensu em Saúde Materno Infantil. Instituto de Medicina Integral Prof. Fernando Figueira. Rua dos Coelhos, 300. Boa Vista. Recife, PE, Brasil. CEP: 50.070-550. E-mail: katzleila@gmail.com

\section{Resumo}

Objetivos: avaliar a adequação do rastreamento e do diagnóstico de diabetes mellitus gestacional em puérperas atendidas em unidade hospitalar de dois municípios da região do Vale do São Francisco.

Métodos: estudo descritivo, com 1340 puérperas que pariram no Hospital Dom Malan-IMIP-PE e Maternidade Municipal de Juazeiro-BA, de abril de 2011 a janeiro de 2012. Excluídas aquelas com diagnóstico prévio de diabetes mellitus, sem pré-natal sem exames acessiveis. Foram construidas tabelas de distribuição de frequência e medidas de tendência central e dispersão.

Resultados: a média de idade gestacional da solicitação da glicemia de jejum foi 14 semanas, adequada em 46\%. A idade gestacional média da realização da glicemia foi 16 semanas, realizada até 30 dias após solicitação em 37,9\%; recebimento do resultado ocorreu em média na $20^{a}$ semana, adequada em 33,4\%. A segunda glicemia de jejum foi solicitada e interpretada adequadamente em 28,1\% dos casos; $20,8 \%$ tiveram resultados glicêmicos da primeira e segunda glicemia de jejum igual ou superior a 85 $m g / d L$ e $11,9 \%$ não realizaram ou receberam a glicemia de jejum. Inadequação no rastreamento/ diagnóstico detectada em 76,9\% $(n=1022)$, e 5,0\% $(n=67)$ de quem realizou Teste Oral de Tolerância a Glicose. Alguma inadequação foi observada em 91,9\% durante o rastreio.

Conclusões: o rastreio/diagnóstico do diabetes gestacional entre as puérperas estudadas foi realizado inadequadamente.

Palavras-chave Diabetes gestacional, Diagnóstico, Avaliação 


\section{Introdução}

O Diabetes Mellitus (DM) relacionado à gravidez pode ser classificado como Diabetes Mellitus prégestacional e Diabetes Mellitus Gestacional (DMG). Caracteriza-se pela intolerância à glicose em graus de intensidade variáveis, com início ou detecção pela primeira vez durante a gestação, podendo ou não, persistir após o parto. ${ }^{1}$

Cerca de $7 \%$ de todas as gestações (variando de $1 \%$ a $14 \%$ dependendo da população estudada e do teste diagnóstico utilizado) são complicadas pelo DMG. No Brasil, a prevalência de DMG é de 7,6\%, sendo considerado um problema de saúde pública. ${ }^{2}$

A presença de diabetes na gestação aumenta o risco materno e acarreta também danos fetais, que podem variar conforme o grau de hiperglicemia e a época em que o distúrbio metabólico ocorre. 3 Mulheres com diabetes apresentam maiores riscos de cesárea, macrossomia e de tocotraumatismo. 4

A presença de DMG também predispõe a mulher a complicações tardias, com uma probabilidade de $30 \%$ de desenvolver Diabetes Mellitus tipo 2, especialmente se permanecerem obesas 5 e de $38 \%$ a $66 \%$ de recidiva de DMG. 4

Atualmente, existem diversos métodos para rastrear e diagnosticar DMG, não havendo consenso sobre qual o melhor esquema. ${ }^{6}$ No Brasil, podem ser utilizados o rastreio e diagnóstico estabelecidos pela Sociedade Brasileira de Diabetes (SBD) 6 e pelo Ministério da Saúde (MS). ${ }^{7}$

Conforme os critérios de rastreio e diagnóstico estabelecidos pelo MS, 7 os quais foram utilizados como referência para a realização desse estudo, preconiza-se a realização da glicemia de jejum (GJ) na primeira consulta pré-natal, independente da presença de fatores de risco.

A GJ com valor igual ou maior que $110 \mathrm{mg} / \mathrm{dL}$, deve ser repetida. Caso esse valor se confirme, considera-se como DMG. Se a GJ for menor do que $85 \mathrm{mg} / \mathrm{dL}$ deve ser repetida na $20^{\mathrm{a}}$ semana. Se continuar menor do que $85 \mathrm{mg} / \mathrm{dL}$ considera-se como rastreio negativo. Valores de GJ entre 85 $\mathrm{mg} / \mathrm{dL}$ e $109 \mathrm{mg} / \mathrm{dL}$ são tidos como o rastreamento positivo, devendo-se solicitar o Teste Oral de Tolerância a Glicose (TOTG) com $75 \mathrm{~g}$ de glicose anidra entre 24 e 28 semanas de gestação. Se o resultado do TOTG for menor do que $110 \mathrm{mg} / \mathrm{dL}$ em jejum e $140 \mathrm{mg} / \mathrm{dL}$ após duas horas considera-se como negativo para DMG, se apresentar valores de jejum maior ou igual que $110 \mathrm{mg} / \mathrm{dL}$ e após duas horas de $140 \mathrm{mg} / \mathrm{dL}$ estabelece o diagnóstico de DMG.7

Apesar da falta de consenso sobre o melhor método para rastreio e diagnóstico do DMGE, ${ }^{8}$ sabese que o sucesso da deteç̧ão do diabetes depende do diagnóstico mais precoce possível, durante a assistência pré-natal, a fim de que a paciente e o feto possam beneficiar-se do tratamento, melhorando os resultados maternos e perinatais. ${ }^{3}$

Visando garantir a qualidade da assistência prénatal conforme política do Programa de Humanização do Pré-natal e Nascimento (PHPN), O MS estabeleceu critérios para avaliação da qualidade da assistência, dentre eles, o número de consultas (seis no mínimo), início de acompanhamento o mais precoce possível e solicitação dos exames complementares obrigatórios. ${ }^{9}$

Apesar de muitas políticas públicas terem sido instituídas, e muitos profissionais se esforçarem para seguir as práticas recomendadas pelos programas de saúde, pouco se avaliou se essa implementação foi adequada ou não. Essa situação pode provocar sérias consequências econômicas e sociais e demandar recursos de saúde, representando um desafio para os gestores, na criação de um sistema de saúde eficiente e eficaz. Diante desse contexto, o objetivo geral do estudo foi avaliar a adequação do rastreamento e do diagnóstico de DMG conforme o protocolo adotado pelo MS em puérperas atendidas em unidade hospitalar de dois municípios da região do Vale do São Francisco.

\section{Métodos}

O estudo foi do tipo descritivo. Foram incluídas puérperas internadas na enfermaria obstétrica dos hospitais Dom Malan-IMIP (HDM) no município de Petrolina, Estado de Pernambuco e Maternidade Municipal do município de Juazeiro, Estado da Bahia. Como critério de exclusão utilizou-se diagnóstico prévio de diabetes mellitus, não ter realizado consulta pré-natal e não ter exames acessíveis. O hosptial Dom Malan é a referência terciária para alto risco na região e atende cerca de 600 partos mês. A Maternidade Municipal do Juazeiro é uma maternidade que atende cerca de 300 partos por mês. $\mathrm{O}$ Dom Malan é o serviço de referência obstétrica de alto risco para a Maternidade de Juazeiro, assim como todos os outros serviços da região.

A amostra foi constituída por 1340 mulheres avaliadas no período de abril de 2011 a janeiro de 2012 (Figura 1). O cálculo da amostra foi realizado com Programa Statcalc, do software de bioestatística de domínio público Epi-info 3.5.3, considerando-se uma frequência de DMG de 7,6\%,5 com um poder de $80 \%$. Estimando-se um erro estatístico de $5 \%$ e um intervalo de confiança de $95 \%$, seriam necessárias 
1116 pacientes. Prevendo-se $20 \%$ de perdas, chegouse a 1339 pacientes.

Durante o período do estudo, ocorreram 7546 partos; foram abordadas 1459 mulheres, sendo que 119 foram excluídas por não atenderem aos critérios de inclusão. A coleta dos dados foi realizada por conveniência, no período da tarde de segunda a sexta-feira e eram abordadas as mulheres que haviam parido no dia anterior. A coleta foi feita pela pesquisadora principal e três assistentes treinados. Durante a entrevista foi preenchido um formulário padronizado com perguntas referentes a dados sociodemográficos e obstétricos, como paridade e número de gestações. Sobre a gestação atual, investigou-se a idade de início do pré-natal, número de consultas realizadas, idade gestacional em que foi solicitada, realizada e recebida a GJ, se houve realização da segunda GJ e do teste diagnóstico TOTG. Para o completo preenchimento dos formulários foi utilizado, além da entrevista à gestante, consulta ao cartão do pré-natal e ao prontuário clínico da mãe e do recém-nascido. Para a avaliação da adequação do rastreamento e do diagnóstico de DMG, foi utilizado como parâmetro o protocolo vigente do MS.7 Considerou-se como adequado quando o início do pré-natal e a solicitação da primeira GJ ocorreram no primeiro trimestre da gestação; quando a realização da GJ ocorreu em 30 dias e o seu recebimento deu-se em 60 dias respectivamente, a partir da data de solicitação da primeira GJ; quando o resultado da GJ foi interpretado conforme necessidade de realizar ou não o teste diagnóstico-TOTG, e no caso de ter realizado o TOTG, se este teve o diagnóstico realizado de forma adequada.

Para análise dos dados utilizou-se o programa Epi-info 3.5.3. Foram construídas tabelas de distribuição de frequência, com os respectivos intervalos de confiança, para as variáveis categóricas, calculando-se ainda, medidas de tendência central e dispersão para as variáveis quantitativas. Os dados somente foram coletados após a aprovação do Comitê de Ética em Pesquisa em seres humanos do Instituto de Medicina Integral Prof. Fernando Figueira (IMIP), sob o n ${ }^{\circ} 2220$.

\section{Figura 1}

Fluxograma de captação das participantes.

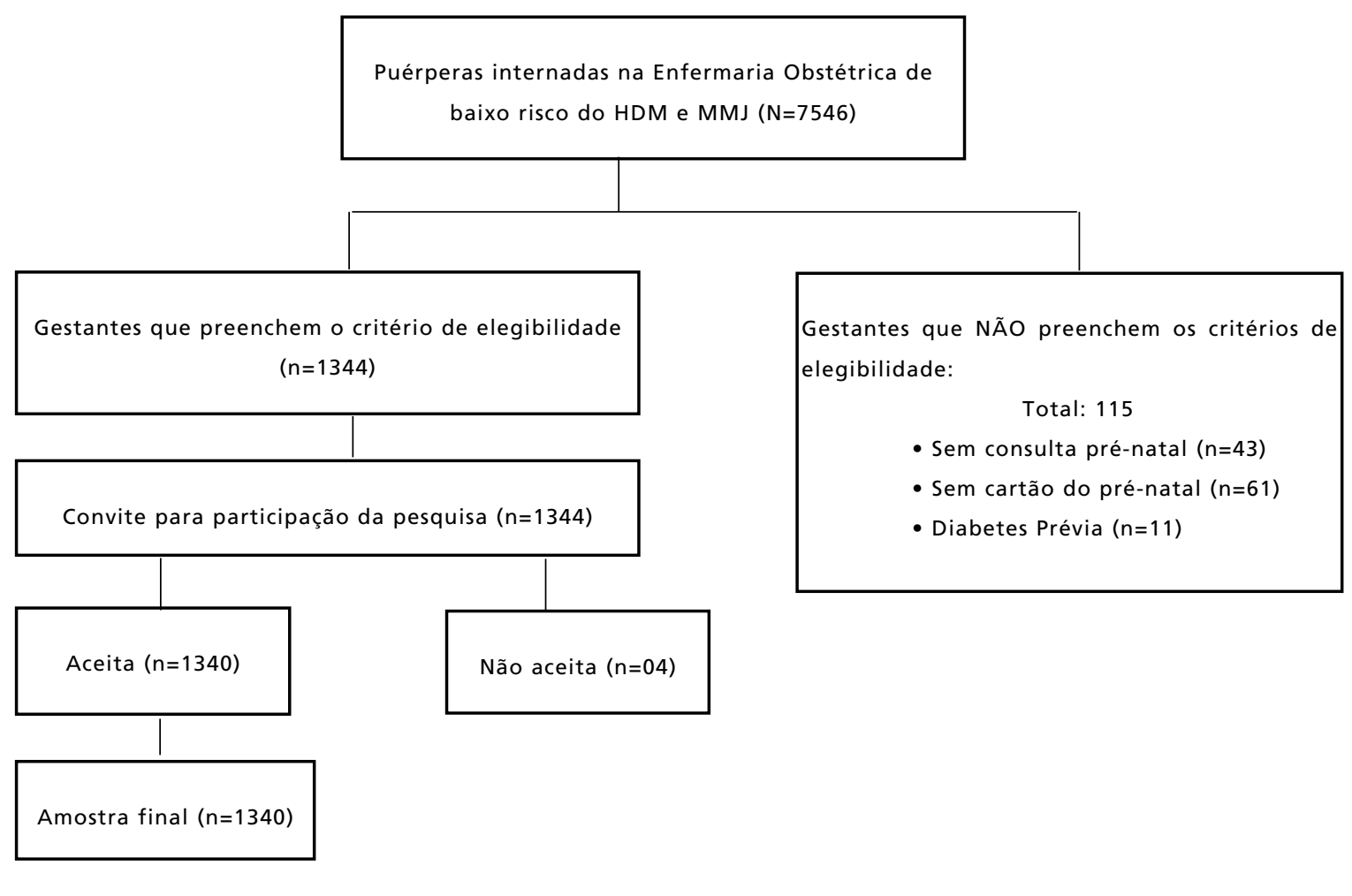

Fonte: Hospital Dom Malan (HDM); Maternidade Municipal de Juazeiro (MMJ); formulários da pesquisa. 


\section{Resultados}

Dentre as 1340 mulheres analisadas, a idade média foi de 24,5 anos ( $\pm 6,4$ anos), a média do Índice de Massa Corporal (IMC) de 23,5 ( $\pm 4,5)$ (Tabela 1). A mediana de gestação foi duas, variando de uma a doze gestações, e a mediana da paridade foi de duas, havendo a variação de um a dez partos (resultado não apresentado em Tabela). A cor predominante foi parda $(75,5 \%)$ e a maioria das mulheres era procedente da zona urbana $63,9 \% ; 41,1 \%$ tinham o ensino fundamental incompleto e apenas $29,3 \%$ concluíram o ensino médio. Pode-se observar que a renda familiar igual a um salário mínimo foi observada em mais da metade da população em estudo $(56,7 \%)$ (dados não apresentados em Tabelas).

Quanto às características do pré-natal e do diagnóstico (Tabela 1), observa-se que a idade gestacional (IG) média de início do pré-natal foi de 14 semanas $( \pm 5,9)$, sendo que $48,6 \%$ iniciaram o prénatal no primeiro trimestre e $51,4 \%$ a partir do segundo trimestre de gestação. $O$ número de consultas no pré-natal apresentou a mediana de sete consultas, com mínimo de uma e máximo de 22 . A IG média em que foi solicitada a glicemia de jejum foi de 14,4 semanas $( \pm 6,2)$, oscilando entre três e 38 semanas; sendo de $46,0 \%$ a frequência de mulheres que tiveram a GJ solicitada na época adequada, ou seja, no primeiro trimestre (Tabela 2).

A IG cuja glicemia de jejum foi realizada teve média de 16,9 semanas $( \pm 6,8)$ e a frequência de mulheres que realizaram a GJ no tempo considerado adequado (trinta dias a partir da solicitação) foi de $37,9 \%$ (Tabela 1). O recebimento do resultado da GJ foi em média 20,2 $( \pm 7,2)$ semanas de gestação (Tabela 1) e a frequência de mulheres que receberam a GJ na época considerada como adequada (sessenta dias a partir da solicitação) foi de $33,4 \%$ (Tabela 2).

Dentre as mulheres que realizaram a primeira GJ, $84,7 \%$ tiveram seu resultado interpretado de forma adequada (Tabela 2), sendo que 18,4\% delas apresentaram a primeira GJ com valor maior ou igual $85 \mathrm{mg} / \mathrm{dL}$. Apenas 27,8\% realizaram a segunda glicemia de jejum (Tabela 1), sendo que apenas

\section{Tabela 1}

Características do pré-natal e do rastreamento para diabetes mellitus gestacional em puérperas atendidas no Hospital Dom Malan - Petrolina/PE e Maternidade Municipal de Juazeiro/BA. Região do Vale do São Francisco, Nordeste do Brasil, 2011-2012.

\begin{tabular}{lc}
\hline Características & \multicolumn{1}{c}{ Variáveis com valores médios } \\
\cline { 2 - 2 } Idade (anos) & $\overline{\mathbf{X}} \pm \mathbf{D P}$ \\
Idade gestacional (semanas) & $24,5 \pm 6,4$ \\
$\quad$ início do pré-natal** & $14,0 \pm 5,9$ \\
da solicitação da 1'a GJ*** & $14,4 \pm 6,2$ \\
da realização da 1a GJ*** & $16,9 \pm 6,8$ \\
do recebimento \# & $20,2 \pm 7,2$ \\
Resultado da 2a GJ & $78,2 \pm 24,3$ \\
Índice de Massa Corporal * & $23,5 \pm 4,5$
\end{tabular}

\begin{tabular}{lc} 
Baixo peso & $124(10,6)$ \\
Peso normal & $681(57,9)$ \\
Sobrepeso & $259(22,0)$ \\
Obesidade & $111(9,5)$ \\
Realização do TOTG & $67(5,0)$ \\
GJ maior que 85 \#\# & $217(18,4)$ \\
Realizou 2a GJ & $372(27,8)$ \\
GJ maior que 85 \#\#\# & $63(16,2)$ \\
Número de consultas (mediana/intervalo interquartil) & $7(5-8)$ \\
\hline
\end{tabular}

GJ = glicemia de jejum; TOTG = Teste Oral de Tolerância a Glicose; *dado disponível para 1175 mulheres; **dado disponível para 1333 mulheres; ${ }^{* * *}$ dado disponível para 1200 mulheres; ${ }^{* * * *}$ dado disponível mulheres para $1177 ;$ \#dado disponível para 1159 mulheres; \#\#dado disponível para 1164 mulheres; \#\#dado disponível para 388 mulheres. 
$28,1 \%$ dessas mulheres tiveram a interpretação desse resultado de forma adequada (Tabela 2). Importante salientar que $16,2 \%$ das mulheres apresentaram um valor da segunda GJ maior ou igual a $85 \mathrm{mg} / \mathrm{dL}$.

Das puérperas investigadas, cerca de $12 \%$ não realizaram, ou não receberam, a GJ. Ao avaliar os resultados da primeira e da segunda GJ, pode-se indicar uma frequência de $20,8 \%$ de mulheres com resultados glicêmicos iguais ou maiores a $85 \mathrm{mg} / \mathrm{dL}$.

Ao se considerar a solicitação, realização e recebimento da GJ, assim com a interpretação de seu resultado, 76,9\% $(n=1022)$ das mulheres apresentaram uma inadequação no decorrer do rastreamento/diagnóstico (Tabela 2), ou seja, não foi solicitada a glicemia de jejum, ou não foi realizada ou não recebeu, ou ainda, pode ter ocorrido falha na interpretação de seu resultado. Apenas $5 \%(n=67)$ destas mulheres realizaram o TOTG (Tabela 1).

Em 91,9\% dos casos ocorreu alguma inadequação para completar o rastreamento. Essa inadequação envolve desde a não solicitação da GJ, ou solicitação fora do prazo estabelecido, até os atrasos de sua rea-lização e recebimento (Tabela 2).

Das mulheres que foram rastreadas/diagnosticadas conforme o valor da glicemia de jejum alterada $(23,7 \%, n=318), 14,7 \%(n=47)$ obteve o diagnóstico de DMG. A partir do diagnóstico, instituiu-se o tratamento: em $11 \%(n=37)$ dietoterapia, em $1,5 \%$ dietoterapia/insulinoterapia $(n=5)$ e $1,5 \%$ $(n=5)$ utilizaram apenas insulinoterapia.

Tabela 2

Adequação do rastreamento/diagnóstico de diabetes mellitus gestacional em puérperas atendidas no Hospital Dom Malan - Petrolina/PE e Maternidade Municipal de Juazeiro/BA. Região do Vale do São Francisco, Nordeste do Brasil, 2011-2012

\begin{tabular}{lcc}
\hline Aspectos & N & $\%$ \\
\hline Adequação da IG de solicitação da 1 a GJ* & 551 & 46,0 \\
Adequação da realização da 1 ${ }^{a}$ GJ ** & 446 & 37,9 \\
Adequação da IG de recebimento da 1a GJ *** & 387 & 33,4 \\
Adequação da interpretação do resultado da 1'aJ **** & 1000 & 34,7 \\
Adequação da solicitação e da interpretação da 2a GJ \# & 327 & 28,1 \\
Glicemia de jejum alterado \#\# & 280 & 20,8 \\
Inadequação do rastreamento/diagnóstico \#\#\# & 1022 & 76,9 \\
Qualquer inadequação no rastreamento/diagnóstico \#\# & 1232 & 91,9 \\
\hline
\end{tabular}

GJ = glicemia de jejum; *dado disponível para 1199 mulheres; ${ }^{* *}$ dado disponível para 1177 mulheres; ${ }^{* * *}$ dado disponível para 1159 mulheres; $* * *$ dado disponível para 1181 mulheres; \#dado disponível para 1164 mulheres; \#\#dado disponível para 1340 mulheres; \#\#dado disponível para 1329 mulheres.

\section{Discussão}

Os resultados deste estudo expressam um rastreio e diagnóstico de DMG inadequados, desde a época da solicitação até a realização e recebimento dos exames, além da interpretação incorreta, não havendo conformidade com o preconizado pelo MS.7

Ao se avaliar as características das mulheres, os dados obtidos remetem a um perfil de mulheres com baixa renda e pouca escolaridade. O achado é esperado na medida em que os hospitais envolvidos são hospitais públicos que atendem exclusivamente pelo Sistema Único de Saúde (SUS).

De forma esperada, a maioria das mulheres procedia da zona urbana. De acordo com o Instituto Brasileiro de Geografia e Estatística (IBGE), 10 a taxa de urbanização no país é de $84 \%$, decorrente da transição demográfica e também nutricional, com isso o processo de urbanização, pode influenciar no estilo de vida contribuindo para a adesão de hábitos de vida e alimentares não saudáveis, levando ao aumento de sobrepeso e obesidade.

Neste estudo a frequência de mulheres com sobrepeso $(22 \%)$ foi elevada, porém, inferior a uma coorte brasileira que acompanhou 924 mulheres ${ }^{11}$ na qual $26,5 \%$ eram obesas. ${ }^{11}$ Dados condizentes com um estudo transversal 12 avalia o impacto da obesidade em idade reprodutiva, onde $19,9 \%$ das mulheres iniciaram a gestação com sobrepeso e $10,6 \%$ tinham DMG. ${ }^{12}$

Esse fato se reveste de importância em decorrência da obesidade ter sido demonstrada como fator preditivo para a necessidade de insulina, ${ }^{11}$ também um dos principais fatores que leva ao desenvolvimento do DMG, uma vez que o excesso de gordura provoca o aumento da resistência à insulina, elevando os níveis de glicose no sangue. 8 
Almejando detectar e prevenir precocemente quaisquer intercorrências, o MS7 orienta a captação da gestante no primeiro trimestre de gestação. No entanto nesse estudo a média de IG de início das consultas de pré-natal foi de 14 semanas, e apenas $48,6 \%$ iniciaram o pré-natal no primeiro trimestre.

Estudos brasileiros mostram resultados discordantes no tocante ao momento do início dessa assistência. Resultados distintos são vistos até mesmo quando comparados diferentes municípios de uma mesma região, no caso o Nordeste, onde se supõe que as mulheres estão expostas as mesmas condições. Numa avaliação de como estava sendo prestada a assistência pré-natal em alguns municípios do Estado do Ceará-Brasil, 13 observou-se que um município apresentou uma captação precoce de $58,2 \%$, enquanto em outro foi evidenciada uma adesão acima de $70 \%$. Em contrapartida, em um outro estudo, utilizando o mesmo desenho metodológico anteriormente citado, que avaliou uma região do interior de São Paulo, observou-se captação mais precoce (cerca de $85 \%$ das mulheres haviam começado o pré-natal precocemente). ${ }^{14}$

Na Região do Vale do São Francisco, no entanto, observou-se a necessidade de intensificação da busca precoce das gestantes, possibilitando o acesso mais rápido ao pré-natal, a fim de garantir o tempo hábil para a implantação das intervenções em saúde, e melhorando a qualidade ao pré-natal.7

A média do número de consultas pré-natais realizadas foi de sete e está em conformidade com a recomendação do MS, o qual defende a realização de no mínimo seis consultas, de forma a garantir uma boa atenção pré-natal. Achados semelhantes foram encontrados por outros estudos brasileiros onde $62,4 \%$ das mulheres envolvidas tiveram de quatro a seis consultas e $18 \%$ mais de sete consultas. 13

Corrobora essa idéia uma coorte de 2741 mães na cidade de Pelotas, RS 15 a qual demonstrou que $77 \%$ das gestantes realizaram seis ou mais consultas, porém o pré-natal mostrou falhas no cumprimento e no manejo das normas de assistência pré-natal, existindo desigualdades nos cuidados oferecidos às mulheres. Ainda sobre a qualidade do pré-natal, os principais problemas apontados na literatura referem-se ao não cumprimento das normas e rotinas por parte dos profissionais, ao não preenchimento de registro e à constatação de que os cuidados dispensados são inversamente direcionados às necessidades. 16

Os resultados do estudo ora em tela apontam para o início tardio de consultas, assim como a não realização dos exames necessários, além da interpre- tação e seguimento incorretos dos exames alterados. Isso demonstra que apesar do número satisfatório de consultas, o pré-natal das gestantes envolvidas apresenta lacunas que podem comprometer o adequado rastreamento e diagnóstico de DMG, fato esse comprovado pela época de solicitação da GJ que nesse estudo foi em média de 14 semanas. É ainda mais grave quando se analisa a realização $(16,9$ semanas) e o recebimento (20,2 semanas) da primeira GJ, época em que a gestante deveria estar realizando a segunda etapa do rastreamento, conforme preconizado pelo MS.7

Ao avaliarmos a adequação no manejo da GJ, percebe-se que há uma redução no número de mulheres que completa toda a etapa do rastreamento de forma correta, a saber, pela frequência de mulheres cuja GJ foi solicitada, realizada e recebida (46\%, 37,9\% e 33,4\%, respectivamente). Esses resultados aproximam-se de uma coorte brasileira realizada com 916 mulheres, em um serviço público, onde $54,1 \%$ realizaram a medida da GJ no primeiro trimestre de gestação.11 É importante enfatizar que a frequência de mulheres que iniciaram pré-natal no primeiro trimestre $(48,6 \%)$ se assemelhou à frequência de mulheres que tiveram a glicemia de jejum solicitada adequadamente. Provavelmente, o início tardio do pré-natal foi o responsável pela solicitação tardia do referido exame, chamando a atenção para a importância da captação dessas mulheres para início do pré-natal no momento correto.

Há que ser considerado também o reduzido número de mulheres que realizaram a avaliação glicêmica pela segunda vez $(27,8 \%)$ corroborando um estudo já citado anteriormente, 14 onde a cobertura de exames básicos a serem realizados no início da gestação foi de $82,6 \%$ caindo para $13,8 \%$ na repetição dos exames preconizados no pré-natal. $\mathrm{O}$ estudo de avaliação nacional do Programa de Humanização do Pré-natal e Nascimento (2004) analisou, por região do país, a realização da segunda bateria de exames complementares. Os seus resultados apontaram para uma reduzida realização desses exames, ficando a Região Nordeste à atrás apenas da Região Norte em inadequação de realização de exames. 17

Esses resultados evidenciam a necessidade de se colocar em prática as normas estabelecidas pelo MS 7 concernentes à assistência pré-natal de qualidade. Para o rastreio de DMG a GJ apresenta-se como uma alternativa simples, prática e de baixo custo. Contudo, observou-se que $11,9 \%$ não realizaram a primeira GJ ou não receberam seu resultado e a maioria $(72,2 \%)$ nem sequer realizou a segunda GJ. 
Constatou-se ainda, uma frequência considerável de resultados glicêmicos da primeira e segunda GJ igual ou superior a $85 \mathrm{mg} / \mathrm{dL}(20,8 \%)$, o que implicaria na realização, segundo o MS,7 de um TOTG. Todavia apenas uma pequena parcela (5\%) realizou o TOTG. De forma semelhante, na coorte de Pelotas, 15 62\% das mulheres também não realizaram o teste diagnóstico para DMG

Esses dados corroboram um estudo realizado com 118 mulheres cuja finalidade foi correlacionar o ganho ponderal e desfecho gestacional, e nenhum caso de DMG foi identificado, sugerindo-se ter havido uma falha no rastreamento e no diagnóstico, durante o acompanhamento pré-natal pelas equipes daquele município. ${ }^{18}$

Apesar de o estudo ter apresentado uma ampla casuística, e de terem sido avaliadas minuciosamente cada passo do rastreamento, o que o reveste de relevância, algumas limitações se deram pelo desenho utilizado na pesquisa. Alguns dados analisados foram coletados através de prontuários, que devido ao preenchimento inadequado, acarretaram informações incompletas. Além disso, parte dos dados era obtida por entrevista, passível de viés de recordação das pacientes.

Outro ponto que merece destaque são os dados oriundos das anotações no cartão do pré-natal. Datas e resultados de exames laboratoriais não eram sempre registrados de maneira adequada, prejudicando a avaliação a respeito da real não solicitação; da não realização, apesar de terem sido solicitados ou se ocorreu sub-registro, o que constitui omissão por parte dos profissionais que assistiram às

\section{Referências}

1. American Diabetes Association. Diagnosis and classification of diabetes mellitus. Diabetes Care. 2010; 33 (Supp1. 1): S62-9.

2. Brasil. Ministério da Saúde. Secretaria de Políticas de saúde. Departamento de Ações Programáticas Estratégicas. Plano de Reorganização da atenção básica á hipertensão arterial e ao diabetes mellitus. Brasília, DF; 2001.

3. Sousa PB, Dias ME. Diabetes mellitus gestacional: diagnóstico e manejo. Brasília Med. 2008; 45: 183-90.

4. Vilar L. Endocrinologia Clínica. 3 ed. Rio de Janeiro: Guanabara Koogan; 2008.

5. Resende J. Obstetrícia fundamental. 9 ed. Rio de Janeiro Guanabara Koogan; 2003.

6. Sociedade Brasileira de Diabetes. Tratamento e acompanhamento de diabetes mellitus - Diretrizes da Sociedade Brasileira de Diabetes. Rio de Janeiro: Diagraphic; 2007.

7. Brasil. Ministério da Saúde. Secretaria de Atenção à Saúde. Departamento de Ações Programáticas Estratégicas. Área Técnica de Saúde da Mulher. Pré-natal e Puerpério: atenção gestantes.

Uma vez que a proposta era avaliar se o rastreamento e o diagnóstico de DMG estavam adequados, foram incluídas mulheres que tinham comprovação da rea-lização do rastreio e por isso as mulheres sem cartão pré-natal não foram investigadas. É possível que nesses casos, possa existir falha na realização dos exames e no registro das informações. Da mesma forma, não foi possível avaliar as mulheres que não realizaram pré-natal.

Isso se constitui numa lacuna da avaliação realizada já que essa parcela de mulheres foi excluída da análise. Um estudo de base populacional19 observou que as pessoas que tem maior necessidade de cuidados de saúde são as que menos os recebem, e que, quando os recebem, são de baixa qualidade, acentuando assim as diferenças, ao invés de corrigir eventuais problemas. Por outro lado, o estudo destaca a elevada frequência de mulheres com GJ alterada e que não realizaram o TOTG indicando que houve um rastreio inadequado, chegando a impossibilitar o diagnóstico da doença. Ainda mais grave é o fato de que a grande maioria, mesmo seguindo os passos do rastreamento, apresentou alguma inadequação, incluindo atrasos nos prazos de recebimento de exames e interpretações inadequadas.

Novos estudos que avaliem os potenciais fatores que levem a falhas do rastreio e diagnóstico de DMG devem ser conduzidos em outras regiões e de forma prospectiva. A identificação desses fatores pode facilitar o desenvolvimento e implementação de ações efetivas com intuito de modificar a presente realidade. qualificada e humanizada. Brasília, DF; 2005.

8. Valladares CG, Komka SB. Prevalência de diabetes mellitus gestacional em gestantes de um centro de saúde de BrasíliaDF. Comun Ciências Saúde. 2008; 19: 11-7.

9. Serruya SJ, Cecatti JG, Lago TG. Programa de humanização no pré-natal e nascimento do Ministério da Saúde no Brasil: resultados iniciais. Cad Saúde Pública. 2004; 20 (5): 1281-

10. IBGE (Instituto Brasileiro de Geografia e Estatística). [acesso em fevereiro 2012]. Disponível em: http/www.ibge.gov.br/home.

11. Detsch JCM, Almeida ACR, Bortolini LGC, Nascimento DJ, Oliveira Jr FC, Réa RR. Marcadores para o diagnóstico e tratamento de 924 gestações com diabetes melito gestacional. Arq Bras Endocrinol Metab. 2011; 55/6: 389-98.

12. Padilha PC, Saunders C, Machado RCM, Silva CL, Bull A, Sally EOF, Accioly E. Associação entre o estado nutricional pré-gestacional e a predição do risco de intercorrências gestacionais. Rev Bras Ginecol Obstet. 2007; 29 (10): 511 
13. Moura ERF, Júnior FH, Rodrigues MSP. Avaliação da assistência pré-natal oferecida em uma microrregião de saúde do Ceará, Brasil. Cad Saúde Pública. 2003; 19 (6): 1791-9.

14. Parada CMGL. Avaliação da assistência pré-natal e puerperal desenvolvidas em uma região do interior do estado de São Paulo em 2005. Rev Bras Saúde Matern Infant. 2008; 8(1): 113-24

15. Rasia ICRB, Albernaz E. Atenção pré-natal na cidade de Pelotas, Rio Grande do Sul, Brasil. Rev Bras Saúde Matern Infant. 2008; 8(4): 401-10.

16. Silveira DS, Santos IS, Costa JSD. Atenção pré-natal na rede básica: uma avaliação da estrutura e do processo. Cad Saúde Pública. 2001; 17 (1): 131-9.

Recebido em 27 de fevereiro de 2013

Versão final apresentada em 17 de setembro de 2013

Aprovado em 26 de dezembro de 2013
17. Brasil. Ministério da Saúde. Secretaria de Ciência, Tecnologia e Insumos Estratégicos. Avaliação nacional do Programa de Humanização do Pré-natal e Nascimento. Rev. Saúde Pública, Brasília, 2008; 42 (2): 383-7.

18. Assunção PL, Melo ASO, Gondim SSR, Benício MHA, Amorim MMR, Cardoso MAA. Ganho ponderal e desfechos gestacionais em mulheres atendidas pelo Programa de Saúde da Família em Campina Grande, PB (Brasil). Rev Bras Epidemiol. 2007; 10 (3): 352-60.

19. Hart JT. The inverse care low. Lancet. 1971; 1: 405-12. 